\title{
A NEW BOUND FOR THE STEINER RATIO
}

\author{
BY
}

\author{
D. Z. DU AND F. K. HWANG
}

\begin{abstract}
Let $V$ denote a given set of $n$ points in the euclidean plane. A Steiner minimal tree for $V$ is the shortest network (clearly, it has to be a tree) interconnecting $V$. Junctions of the network which are not in $V$ are called Steiner points (those in $V$ will be called regular points). A shortest tree interconnecting $V$ without using any Steiner points is called a minimal tree. Let $\sigma(V)$ and $\mu(V)$ denote the lengths of a Steiner minimal tree and a minimal tree, respectively. Define $\rho$ to be the greatest lower bound for the ratio $\sigma(V) / \mu(V)$ over all $V$. We prove $\rho>.8$.
\end{abstract}

1. Introduction. Let $V$ denote a given set of $n$ points in the euclidean plane. A Steiner minimal tree for $V$ is the shortest network (clearly, it has to be a tree) interconnecting $V$. Junctions of the network which are not in $V$ are called Steiner points (those in $V$ will be called regular points). A shortest tree interconnecting $V$ without using any Steiner points is called a minimal tree. Let $\sigma(V)$ and $\mu(V)$ denote the lengths of a Steiner minimal tree and a minimal tree, respectively. Define $\rho$ to be the greatest lower bound for the ratio $\sigma(V) / \mu(V)$ over all $V$. Gilbert and Pollak [2] conjectured that $\rho=\sqrt{3} / 2 \cong .866$ and proved its truth for $n=3$. Pollak [5] proved the conjecture for the case $n=4$. For general $n$, Morse (see [3]) proved that $\rho \geqslant .5$, Graham and Hwang [3] proved that $\rho \geqslant 1 / \sqrt{3}$, Chung and Hwang [1] proved that $\rho \geqslant[2 \sqrt{3}+2-(7+2 \sqrt{3})]^{1 / 2} \cong .743$. In this paper we give an elementary proof that $\rho>.8$.

2. Some preliminary results. Let $d(u, v)$ denote the euclidean distance between two points $u$ and $v$, and for a set $X$ of line segments let $L_{X}$ denote the sum of their lengths.

We first observe that in proving a lower bound for $\rho$ we can restrict our attention to Steiner minimal trees in which all regular points have degree one. For, if we know that $\rho \geqslant \alpha$ for all such trees, then $\rho \geqslant \alpha$ will follow in general, because any Steiner tree with regular points of degree greater than one can be broken at such points to form a collection of smaller trees in which all regular points have degree exactly one.

We now prove two technical lemmas that will be applied repeatedly in proving the main theorem. The following simple lemma from [1] will be useful in proving the first of these:

LEMMA 1 [1]. In a Steiner minimal tree $S$, let $A$ be a regular point of degree 1 adjacent to a Steiner point $s$. Let $Y$ be any point on a line of $S$ other than the line $[A, s]$. Then it must be the case that $d(A, Y) \geqslant d(A, s)$.

Received by the editors January 22, 1982.

1980 Mathematics Subject Classification. Primary 51M15; Secondary 05 C05.

(C)1983 American Mathematical Society $0002-9947 / 82 / 0000-0345 / \$ 02.75$ 
Consider a subtree of the Steiner minimal tree $S$ consisting of lines $[A, s],[s, r]$ and $[r, P]$ (see Figure 1), where $A$ is a regular point, $s$ and $r$ are Steiner points, $P$ is either a Steiner point or a regular point, $d(A, s) \geqslant d(r, P),[A, X]$ is perpendicular to $[P, X]$, and $\Varangle A s r=\Varangle s r P=\Varangle r P X=120^{\circ}$ (the three lines at a Steiner point in a Steiner minimal tree always form $120^{\circ}$ angles [2]). Note that this implies that $[A, s]$ is parallel to $[P, X]$. Let the region $A s^{\prime} r^{\prime} P^{\prime} X$ be the mirror reflection of the region $A \operatorname{sr} P X$ with respect to $[A, X]$.

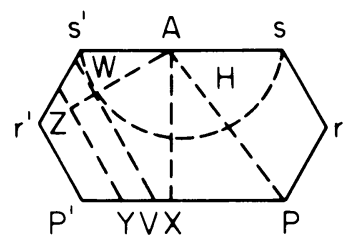

FIGURE 1. A trapping region

LEMMA 2. There must exist a regular point other than $A$, and connected to $A$ through $s$, in the region $A s r P X P^{\prime} r^{\prime} s^{\prime}$.

Proof. Consider the half-circle $H$ with center $A$ and radius $[A, s]$. By Lemma 1 , no line of $S$ can intersect the interior of $H$. If $P$ is a regular point, we are through; otherwise $S$ has a line $[P, Y]$ in the direction $\left[P, P^{\prime}\right]$. Note that $\Varangle A s r=\Varangle s r P$ and $d(A, s) \geqslant d(r, P)$ together imply that $\Varangle r P A \geqslant \Varangle P A s$. Furthermore, since $\Varangle r P A$ $+\Varangle P A s=120^{\circ}$, it follows that $\Varangle A P P^{\prime}=\Varangle P A s \leqslant 60^{\circ}$. If $d(P, Y)>d\left(P, P^{\prime}\right)$, then $\Varangle Y A P>\Varangle P^{\prime} A P=180^{\circ}-2 \Varangle A P P^{\prime} \geqslant 60^{\circ} \geqslant \Varangle A P Y$. Hence $d(P, Y)>$ $d(A, Y)$, and we can shorten the tree by replacing $[P, Y]$ by $[A, Y]$, contradicting the minimality of $S$. Thus we must have $d(P, Y) \leqslant d\left(P, P^{\prime}\right)$.

Let $V$ be the point on $\left[P, P^{\prime}\right]$ such that $[V, W]$ is tangent to $H$, meeting $H$ at $W$, and $\Varangle P V W=120^{\circ}$. Then $\Varangle A W V=90^{\circ}$. Furthermore $\Varangle W A P \geqslant 90^{\circ}$ since $\Varangle A P V \leqslant 60^{\circ}$. It follows $d(P, V)>d(A, W)$.

If $d(P, Y)<d(P, V)$, then we are done, since the fact that every Steiner point must have exactly three incident lines, at angles of $120^{\circ}$, implies that $Y$ must start a path trapped in the region $A s r P X P^{\prime} r^{\prime} s^{\prime}$ minus $H$ (because the path can never enter $H)$ and that path must eventually end at a regular point within the required region.

If $d(P, Y) \geqslant d(P, V)$, with $Y$ lying between $V$ and $P^{\prime}$, then the upward line from $Y$ parallel to $[s, r]$ must cross over $\left[s^{\prime}, r^{\prime}\right]$, or it would again start a path trapped in the specified region. Let $[A, Z]$ be perpendicular to this upward line, meeting it at $Z$. Then $W$ is on $[A, Z]$ and

$$
d(P, Y)=d(P, V)+d(V, Y)>d(A, W)+d(W, Z)=d(A, Z) .
$$

Hence we can shorten the tree by replacing $[P, Y]$ by $[A, Z]$, contradicting the minimality of $S$, and completing the proof.

COROllaRY. Let $q$ be an arbitrary point in the region AsrPXP' $r^{\prime} s^{\prime}$. Suppose that $d(s, r)+d(r, P) \leqslant d(A, s)$. Then

$$
d(A, q) \leqslant d(A, r)=\left[d(A, s)^{2}+d(A, s) d(s, r)+d(s, r)^{2}\right]^{1 / 2} .
$$


Suppose that $d(s, r)+d(r, P) \geqslant d(A, s)$. Then

$$
\begin{aligned}
d(A, q) \leqslant d(A, P)=\left\{[d(A, s)-d(r, P)]^{2}+[d(A, s)-d(r, P)]\right. & \\
\left.\cdot[d(s, r)+d(r, P)]+[d(s, r)+d(r, P)]^{2}\right\}^{1 / 2} & .
\end{aligned}
$$

Our second technical lemma takes a form that is particularly useful in induction proofs. Let $S$ be a Steiner minimal tree for the set of regular points $V$. Regarding $S$ simply as a collection of points in the plane, let $S$ be partitioned into two sets $S^{\prime}$ and $S-S^{\prime}$. Let $t$ be a graph such that $t \cup\left\{S-S^{\prime}\right\}$ is a tree interconnecting $V$. Suppose the lines of $t$ can be partitioned into $t^{*}$ (with vertex set $V^{*} \subseteq V$ ) and $t-t^{*}$ at a regular point so that $t^{*}$ is nonempty and $\left(t-t^{*}\right) \cup\left(S-S^{\prime}\right)$ is a tree interconnecting $V-\left\{V \cap S^{\prime}\right\}$. Define $\rho^{\prime}=\left(L_{S^{\prime}}-L_{t-t^{*}}\right) / L_{t^{*}}$.

Lemma 3. Suppose for some $\alpha$ that $\sigma(V) / \mu(V)>\alpha$ has been proved for all $V$ with $|V|<n$. Let $S$ be a Steiner minimal tree for a set $V$ with $|V|=n$. Then, if there exist choices for $S^{\prime}, t$, and $t^{*}$ as above such that $\rho^{\prime}>\alpha$, we must have $\sigma(V) / \mu(V)>\alpha$.

Proof.

$$
\begin{aligned}
\sigma(V) & =L_{S^{\prime}}+L_{S-S^{\prime}}>\alpha L_{t^{*}}+L_{t-t^{*}}+L_{S-S^{\prime}} \\
& >\alpha L_{t^{*}}+\sigma\left(V-\left\{V \cap S^{\prime}\right\}\right) \\
& >\alpha L_{t^{*}}+\alpha \cdot \mu\left(V-\left\{V \cap S^{\prime}\right\}\right) \geqslant \alpha \cdot \mu(V)
\end{aligned}
$$

where the first inequality follows by assumption, the second is due to the fact that $\left(t-t^{*}\right) \cup\left(S-S^{\prime}\right)$ is a tree interconnecting $V-\left\{V \cap S^{\prime}\right\}$, the third follows from the "inductive" assumption of the lemma, and the last follows because the union of $t^{*}$ and a minimal tree for $V-\left\{V \cap S^{\prime}\right\}$ must be a tree interconnecting $V$ and having no Steiner point.

We quote a result from [2].

LEMMA 4. Suppose that $s_{1}$ and $s_{2}$ are two Steiner points which are connected by a line in the Steiner minimal tree. $s_{1}$ and $s_{2}$ each have two other lines. Let the shortest of these four lines have length $l$. Then $d\left(s_{1}, s_{2}\right) \geqslant(\sqrt{3}-1) l$.

Finally, we give a result which helps recognize a monotone increasing function. The proof makes use of the fact $\left(b^{2}+b x+x^{2}\right)^{1 / 2}>x+b / 2$ which is also frequently used elsewhere.

LEMMA 5. Let

$$
f(x)=\frac{c+x}{a+\left(b^{2}+b x+x^{2}\right)^{1 / 2}}
$$

where $a, b$ and $c$ are constants and $b>0$. Then $f(x)$ is monotone increasing in $x$ if $2 a+b-2 c \geqslant 0$. 
Proof. Let $N(d f / d x)$ be the numerator of $d f / d x$. Since we are only interested in the sign of $d f / d x$, it suffices to check the sign of $N(d f / d x)$ :

$$
\begin{aligned}
N\left(\frac{d f}{d x}\right) & =a+\left(b^{2}+b x+x^{2}\right)^{1 / 2}-(c+x) \frac{b+2 x}{2\left(b^{2}+b x+x^{2}\right)^{1 / 2}} \\
& =\frac{2 a\left(b^{2}+b x+x^{2}\right)^{1 / 2}+2\left(b^{2}+b x+x^{2}\right)-(c+x)(b+2 x)}{2\left(b^{2}+b x+x^{2}\right)^{1 / 2}} \\
& >\frac{2 a(x+b / 2)+2 b^{2}+b x-b c-2 c x}{2\left(b^{2}+b x+x^{2}\right)^{1 / 2}} \\
& =\frac{b(a+2 b-c)+(2 a+b-2 c) x}{2\left(b^{2}+b x+x^{2}\right)^{1 / 2}}>0 .
\end{aligned}
$$

\section{The main result.}

THEOREM. $\rho>.8$.

Proof. Previous results [5] have shown that $\sigma(V) / \mu(V) \geqslant \sqrt{3} / 2>.8$ for all sets $V$ with $|V| \leqslant 4$. Thus, suppose that $\sigma(V) / \mu(V)>.8$ for all sets $V$ with $|V|<n$, and consider any set $V$ with $|V|=n \geqslant 5$ and Steiner minimal tree $S$. Let $s$ be a Steiner point in $S$ adjacent to two regular points $A$ and $B$ and a Steiner point $r$ that is adjacent to two other points $P$ and $Q$ (see Figure 2). Such a configuration must exist in $S$, since we can assume that $n>4$ and that all regular points in $S$ have degree 1 .

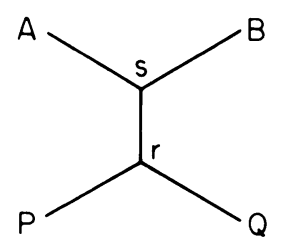

Figure 2. A subtree of $S$

Without loss of generality, assume $d(B, s)=w \geqslant d(A, s)=1$. We also label $d(s, r)$ by $\theta, d(r, P)$ by $x$ and $d(r, Q)$ by $y$ to simplify writing. We partition the proof into two parts, each having several subcases.

Part 1. $x \leqslant 1$. Let $[P, X]$ be parallel to $[A, s]$ and perpendicular to $[A, X]$. Let $R$ denote the region of $A \operatorname{sr} P X$ together with its minor image with respect to $[A, X]$. By Lemma 2 there exists a regular point $C$ in $R$.

Case (i). $\theta \geqslant 1$. Set $S^{\prime}=[A, s] \cup[B, s] \cup[s, r], t-t^{*}=\varnothing$ and $t^{*}=[A, B] \cup$ $[A, C]$. By the Corollary of Lemma 2 ,

$$
d[A, C] \leqslant 1+\theta .
$$

Hence

$$
\rho^{\prime} \geqslant \frac{1+w+\theta}{\left(1+w+w^{2}\right)^{1 / 2}+1+\theta}=g_{1}(w, \theta)
$$


which is monotone increasing in $w$ by Lemma 5 and is also easily verified to be monotone increasing in $\theta$. Therefore

$$
\rho^{\prime} \geqslant g_{1}(1,1)=\frac{3}{3^{1 / 2}+2}>.8 \text {. }
$$

Case (ii). $\theta \leqslant 1$ and $x \leqslant 1-\theta$. By the Corollary of Lemma 2 ,

$$
d(A, C) \leqslant d(A, r)=\left(1+\theta+\theta^{2}\right)^{1 / 2} .
$$

If $\left(1+\theta+\theta^{2}\right)^{1 / 2}<1.25$, set $S^{\prime}=[A, s], t-t^{*}=\varnothing$ and $t^{*}=[A, C]$. Then

$$
\rho^{\prime}>\frac{1}{1.25}=.8 \text {. }
$$

If $\left(1+\theta+\theta^{2}\right)^{1 / 2} \geqslant 1.25$, set $S^{\prime}=[A, s] \cup[B, s] \cup[s, r], t-t^{*}=\varnothing$ and $t^{*}=$ $[A, B] \cup[A, C]$. Then

$$
\rho^{\prime} \geqslant \frac{1+w+\theta}{\left(1+w+w^{2}\right)^{1 / 2}+\left(1+\theta+\theta^{2}\right)^{1 / 2}}=g_{2}(w, \theta)
$$

which is monotone increasing in $\theta$ by Lemma 5 and is also easily seen to be monotone increasing in $w$. Let $\theta^{*}=\left(13^{1 / 2}-2\right) / 4(>.4)$ be the unique positive solution of the equation $\left(1+\theta+\theta^{2}\right)^{1 / 2}=1.25$. Then

$$
\rho^{\prime} \geqslant g_{2}\left(1, \theta^{*}\right)=\frac{2+\theta^{*}}{3^{1 / 2}+\left(1+\theta^{*}+\left(\theta^{*}\right)^{1 / 2}\right)}>.8 .
$$

Case (iii). $\theta \leqslant 1,1-\theta \leqslant x \leqslant 1$ and $1-\theta \leqslant y$. Let $S^{\prime}=[A, s] \cup[B, s] \cup[s, r]$ $\cup[r, P] \cup[r, Q], t-t^{*}=[P, Q]$ and $t^{*}=[A, B] \cup[A, C]$. Then

$$
\rho^{\prime} \geqslant \frac{1+w+\theta+x+y-\left(x^{2}+x y+y^{2}\right)^{1 / 2}}{\left(1+w+w^{2}\right)^{1 / 2}+1+\theta}
$$

which is easily verified to be monotone increasing in $x$ and in $y$. Hence, setting $x=1-\theta$ and $y=1-\theta$, we obtain

$$
x+y-\left(x^{2}+x y+y^{2}\right)^{1 / 2} \geqslant\left(2-3^{1 / 2}\right)(1-\theta) .
$$

It follows

$$
\rho^{\prime} \geqslant \frac{3-3^{1 / 2}+w+\left(3^{1 / 2}-1\right) \theta}{\left(1+w+w^{2}\right)^{1 / 2}+1+\theta}=g_{3}(w, \theta)
$$

which is monotone increasing in $w$ by Lemma 5 . Hence

$$
\rho^{\prime} \geqslant g_{3}(1, \theta)=\frac{4-3^{1 / 2}+\left(3^{1 / 2}-1\right) \theta}{3^{1 / 2}+1+\theta}
$$

which is easily verified to be monotone decreasing in $\theta$. Therefore,

$$
\rho^{\prime} \geqslant g_{3}(1,1)=\frac{3}{3^{1 / 2}+2}>.8 \text {. }
$$


Case (iv). $\theta \leqslant 1,1-\theta \leqslant x \leqslant 1$ and $y \leqslant 1-\theta$. Let $R^{\prime}$ be the region similarly defined as $R$ except it lies on the right side of $[s, r]$. Since $y \leqslant w$, there exists a regular point $C^{\prime}$ in $R^{\prime}$. Let $S^{\prime}=[A, s]+[B, s]+[s, r], t-t^{*}=\varnothing$ and $t^{*}=$ $[A, C] \cup\left[B, C^{\prime}\right]$. By the Corollary of Lemma 2 ,

$$
d[A, C] \leqslant 1+\theta, \quad d\left[B, C^{\prime}\right] \leqslant\left(w^{2}+w \theta+\theta^{2}\right)^{1 / 2} .
$$

Hence

$$
\rho^{\prime} \geqslant \frac{1+w+\theta}{1+\theta+\left(w^{2}+w \theta+\theta^{2}\right)^{1 / 2}}=g_{4}(w, \theta)
$$

which is monotone increasing in $w$ by Lemma 5 . Therefore,

$$
\rho \geqslant g_{4}(1, \theta)=\frac{2+\theta}{1+\theta+\left(1+\theta+\theta^{2}\right)^{1 / 2}}
$$

which is easily verified to be monotone decresing in $\theta$. It follows

$$
\rho \geqslant g_{4}(1,1)=\frac{3}{2+3^{1 / 2}} \geqslant .8 .
$$

Part 2. $x \geqslant 1$. Let $P^{*}$ denote the point on $[r, P]$ such that $d\left[r, P^{*}\right]=1$. Let $T$ denote the Steiner minimal tree on the three points $A, P^{*}$ and $Q$. Let $\triangle W A P^{*}$ be an equilateral triangle such that $W$ and $r$ lie on opposite sides of $\left[A, P^{*}\right]$. Then the length of $T$ equals $d(W, Q)$ by Melzak's construction (see Figure 3 ).

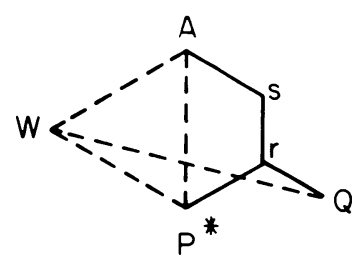

FIGURE 3. Melzak's construction for $T$

LEMMA 6. The length of $T$ is $\left[(\theta+y)^{2}+3(\theta+y)+3\right]^{1 / 2}$.

Proof. Let $a$ denote the angle $\Varangle r P^{*} Q$. Then

$$
\sin a=\frac{y \sin 120^{\circ}}{d\left(P^{*}, Q\right)}=\frac{3^{1 / 2} y}{2\left(1+y+y^{2}\right)^{1 / 2}} .
$$


It follows

$$
\begin{aligned}
& \cos a=\frac{y+2}{2\left(1+y+y^{2}\right)^{1 / 2}} . \\
& \begin{aligned}
d[W, Q] & =\left[d\left(W, P^{*}\right)^{2}+d\left(P^{*}, Q\right)^{2}-2 \cos \left(120^{\circ}+a\right) d\left(W, P^{*}\right) d\left(P^{*}, Q\right)\right]^{1 / 2} \\
& =\left[(1+\theta)^{2}+1+y+y^{2}+2 \cos \left(60^{\circ}-a\right)(1+\theta)\left(1+y+y^{2}\right)^{1 / 2}\right]^{1 / 2} \\
& =\left[(1+\theta)^{2}+1+y+y^{2}\right. \\
& \left.+2\left(\cos 60^{\circ} \cos a+\sin 60^{\circ} \sin a\right)(1+\theta)\left(1+y+y^{2}\right)^{1 / 2}\right]^{1 / 2} \\
& =\left[(1+\theta)^{2}+1+y+y^{2}\right. \\
& \left.+(2 y+1)\left(1+y+y^{2}\right)^{-1 / 2}(1+\theta)\left(1+y+y^{2}\right)^{1 / 2}\right]^{1 / 2} \\
= & {\left[(\theta+y)^{2}+3(\theta+y)+3\right]^{1 / 2} . }
\end{aligned}
\end{aligned}
$$

For all subcases of Part 2, $S^{\prime}$ is set to be $[A, s] \cup[B, s] \cup[s, r] \cup\left[r, P^{*}\right] \cup[r, Q]$.

Case (i). $y>1$. By Lemma $4, \theta \geqslant 3^{1 / 2}-1$. Let $Q^{*}$ denote the point on $[r, Q]$ such that $d\left[r, Q^{*}\right]=1$. Let $T^{*}$ denote the Steiner minimal tree on the three points $A, P^{*}$ and $Q^{*}$. By Lemma 6 , the length of $T^{*}$ is

$$
\left[(\theta+1)^{2}+3(\theta+1)+3\right]^{1 / 2}=\left(\theta^{2}+5 \theta+7\right)^{1 / 2} .
$$

Set $t-t^{*}=T^{*}$ and $t^{*}=[A, B]$. Then

$$
\rho^{\prime}=\frac{3+w+\theta-\left(\theta^{2}+5 \theta+7\right)^{1 / 2}}{\left(1+w+w^{2}\right)^{1 / 2}}=f_{1}(w, \theta)
$$

which is monotone increasing in $w$ by Lemma 6 and is also easily verified to be monotone increasing in $\theta$. Therefore

$$
\rho^{\prime} \geqslant f_{1}\left(1,3^{1 / 2}-1\right)=\frac{3+3^{1 / 2}-\left(6+3 \cdot 3^{1 / 2}\right)^{1 / 2}}{3^{1 / 2}}>.8 .
$$

We assume $y \leqslant 1$ in all other cases. Furthermore, there exists a regular point $C^{\prime}$ in the region $R^{\prime}$. We also define $u=\theta+y$. By Lemma $4, u \geqslant 3^{1 / 2} y$.

Case (ii). $u \leqslant .95$ or $.95 \leqslant u \leqslant 1$ and $w \geqslant 1.11$. Set $t-t^{*}=T$ and $t^{*}=\left[B, C^{\prime}\right]$. Then

$$
d\left(B, C^{\prime}\right) \leqslant\left[w^{2}+(u-y) w+(u-y)^{2}\right]^{1 / 2}
$$

and

$$
\begin{aligned}
\rho^{\prime} & \geqslant \frac{2+w+u-\left(u^{2}+3 u+3\right)^{1 / 2}}{\left[w^{2}+(u-y) w+(u-y)^{2}\right]^{1 / 2}} \\
& \geqslant \frac{2+w+u-\left(u^{2}+3 u+3\right)^{1 / 2}}{\left(u^{2}+w u+w^{2}\right)^{1 / 2}}=f_{2}(u, w) ;
\end{aligned}
$$




$$
\begin{aligned}
N\left(\frac{\partial f_{2}}{\partial u}\right)= & \left(w^{2}+w u+u^{2}\right)^{1 / 2}\left[1-\frac{2 u+3}{2\left(u^{2}+3 u+3\right)^{1 / 2}}\right] \\
& -\left[2+w+u-\left(u^{2}+3 u+3\right)^{1 / 2}\right] \frac{2 u+w}{2\left(u^{2}+w u+w^{2}\right)^{1 / 2}} .
\end{aligned}
$$

Multiplying through by $2\left(u^{2}+3 u+3\right)^{1 / 2}\left(u^{2}+w u+w^{2}\right)^{1 / 2}$, then the right side becomes

$$
\begin{aligned}
{\left[2\left(u^{2}+w u+w^{2}\right)-(2+\right.} & w+u)(2 u+w)]\left(u^{2}+3 u+3\right)^{1 / 2} \\
& +\left(u^{2}+3 u+3\right)(2 u+w)-\left(u^{2}+w u+w^{2}\right)(2 u+3) \\
= & -[(4+w) u+w(2-w)]\left(u^{2}+3 u+3\right)^{1 / 2} \\
& +(3-w) u^{2}+2\left(3-w^{2}\right) u+3 w(1-w) \\
< & -[(4+w) u+2 w](u+1.5)+7^{1 / 2} w^{2} \\
& +(3-w) u^{2}+2\left(3-w^{2}\right) u+3 w(1-w) \\
= & -\left[(1+2 w) u^{2}+(3.5+2 w) w u+3-7^{1 / 2} w^{2}\right]<0 .
\end{aligned}
$$

Therefore, it suffices to prove

$$
f_{2}(.95, w)>.8 \text { for } u \leqslant .95 \text { and } w \geqslant 1
$$

and

$$
f_{2}(1, w)>.8 \text { for } .95 \leqslant u \leqslant 1 \text { and } w \geqslant 1.11 \text {. }
$$

It is easily verified by Lemma 5 that $f_{2}(.95, w)$ and $f_{2}(1, w)$ are both monotone increasing in $w$. It follows, for $u \leqslant .95$ and $w \geqslant 1$,

$$
\rho^{\prime} \geqslant f_{2}(.95,1)=\frac{3.95-6.7525^{1 / 2}}{2.8525^{1 / 2}}>\frac{3.95-2.598}{1.69}>.8 .
$$

For $.95 \leqslant u \leqslant 1$ and $w \geqslant 1.11$,

$$
\rho^{\prime} \geqslant f_{2}(1,1.11)=\frac{4.11-7^{1 / 2}}{3.3421^{1 / 2}}>\frac{4.11-2.65}{1.829}>.8 .
$$

Case (iii). $u \geqslant 1$ and $w \geqslant 1.11$. Set $t-t^{*}=T$ and $t^{*}=[A, B]$. Then

$$
\rho^{\prime}=\frac{2+w+u-\left(u^{2}+3 u+3\right)^{1 / 2}}{\left(1+w+w^{2}\right)^{1 / 2}}=f_{3}(u, w)
$$

which is monotone increasing in $w$ by Lemma 5 and is also easily verified to be monotone increasing in $u$. Therefore

$$
\rho^{\prime} \geqslant f_{3}(1,1.11)=f_{2}(1,1.11)>.8 \text {. }
$$

Case (iv). $.95 \leqslant u$ and $w \leqslant 1.11$. Set $t-t^{*}=\left[P^{*}, Q\right]$ and $t^{*}=[A, B] \cup\left[B, C^{\prime}\right]$.

Subcase (a). $u \leqslant w$. Then

$$
d\left[B, C^{\prime}\right] \leqslant\left[w^{2}+(u-y) w+(u-y)^{2}\right]^{1 / 2}
$$


and

$$
\begin{aligned}
\rho^{\prime} \geqslant & \frac{2+w+u-\left(1+y+y^{2}\right)^{1 / 2}}{\left(1+w+w^{2}\right)^{1 / 2}+\left[w^{2}+(u-y) w+(u-y)^{2}\right]^{1 / 2}}=f_{4}(u, w, y) ; \\
N\left(\frac{\partial f_{4}}{\partial w}\right)= & \left(1+w+w^{2}\right)^{1 / 2}+\left[w^{2}+(u-y) w+(u-y)^{2}\right]^{1 / 2} \\
& -\left[2+w+u-\left(1+y+y^{2}\right)^{1 / 2}\right] \\
& \cdot\left[\frac{1+2 w}{2\left(1+w+w^{2}\right)^{1 / 2}}+\frac{2 w+u-y}{2\left[w^{2}+(u-y) w+(u-y)^{2}\right]^{1 / 2}}\right] \\
< & \left(1+w+w^{2}\right)^{1 / 2}+\left[w^{2}+(u-y) w+(u-y)^{2}\right]^{1 / 2}-(1+w+u-y) \\
& \cdot\left[\frac{1+2 w}{2\left(1+w+w^{2}\right)^{1 / 2}}+\frac{2 w+u-y}{2\left[w^{2}+(u-y) w+(u-y)^{2}\right]^{1 / 2}}\right] \\
< & 0
\end{aligned}
$$

since

$$
\frac{2\left(1+w+w^{2}\right)-(1+w+u-y)(1+2 w)}{2\left(1+w+w^{2}\right)^{1 / 2}}<0
$$

and

$$
\begin{gathered}
\frac{2\left[w^{2}+(u-y) w+(u-y)^{2}\right]-(1+w+u-y)(2 w+u-y)}{2\left[w^{2}+(u-y) w+(u-y)^{2}\right]^{1 / 2}} \\
=\frac{-2 w-(1+w)(u-y)+(u-y)^{2}}{2\left[w^{2}+(u-y) w+(u-y)^{2}\right]^{1 / 2}}<0 .
\end{gathered}
$$

Furthermore,

$$
\begin{aligned}
N\left(\frac{\partial f_{4}}{\partial u}\right)= & \left(1+w+w^{2}\right)^{1 / 2}+\left[w^{2}+(u-y) w+(u-y)^{2}\right]^{1 / 2} \\
& -\left[2+w+u-\left(1+y+y^{2}\right)^{1 / 2}\right] \frac{w+2(u-y)}{2\left[w^{2}+(u-y) w+(u-y)^{2}\right]^{1 / 2}} \\
> & \left(1+w+w^{2}\right)^{1 / 2}+\left[w^{2}+(u-y) w+(u-y)^{2}\right]^{1 / 2} \\
& -\left[2+w+u-\left(1+y+y^{2}\right)^{1 / 2}\right] \\
> & 1+w / 2+w+(u-y) / 2-(2+w+u)+1+y / 2 \\
= & (w-u) / 2 \geqslant 0 .
\end{aligned}
$$


Since $u \geqslant \max \left\{.95,3^{1 / 2} y\right\}$, it suffices to show

$$
f_{4}(.95,1.11, y)>.8 \text { for } 3^{1 / 2} y \leqslant .95
$$

and

$$
f_{4}\left(3^{1 / 2} y, 1.11, y\right)>.8 \text { for } .95 \leqslant 3^{1 / 2} y \leqslant w .
$$

We first show that for $y \leqslant .95 / 3^{1 / 2}<.55$,

$$
f_{4}(.95,1.11, y)=\frac{4.05-\left(1+y+y^{2}\right)^{1 / 2}}{3.3421^{1 / 2}+\left(y^{2}-3.01 y+3.1891\right)^{1 / 2}}>.8 .
$$

Note that the numerator and the denominator are both monotone decreasing in $y$ for $0 \leqslant y \leqslant 1$. If for $y \leqslant y \leqslant \bar{y}$ we set $y=\bar{y}$ in the numerator and set $y=y$ in the denominator and the ratio is still greater than .8 , then $f_{4}(.95,1.11, y)>.8$ for $\underline{y} \leqslant y \leqslant \bar{y}$. For $0 \leqslant y \leqslant .2$,

$$
f_{4}(.95,1.11, y)>\frac{4.05-1.24^{1 / 2}}{1.83+3.1891^{1 / 2}}>\frac{4.05-1.12}{1.83+1.79}>.8
$$

For $.2 \leqslant y \leqslant .4$

$$
f_{4}(.95,1.11, y)>\frac{4.05-1.56^{1 / 2}}{1.83+2.6271^{1 / 2}}>\frac{4.05-1.25}{1.83+1.63}>.8 \text {. }
$$

For $.4 \leqslant y \leqslant .55$,

$$
f_{4}(.95,1.11, y)>\frac{4.05-1.8525^{1 / 2}}{1.83+2.1451^{1 / 2}}>\frac{4.05-1.33}{1.83+1.47}>.8 .
$$

Next we show that for $.54<.95 / 3^{1 / 2} \leqslant y \leqslant 1.11 / 3^{1 / 2}<.641$,

$$
\begin{aligned}
f_{4}\left(3^{1 / 2} y, 1.11, y\right) & =\frac{3.11+3^{1 / 2} y-\left(1+y+y^{2}\right)^{1 / 2}}{3.3421^{1 / 2}+\left[\left(4-2 \cdot 3^{1 / 2}\right) y^{2}+1.11\left(3^{1 / 2}-1\right) y+1.2321\right]^{1 / 2}} \\
& >.8 .
\end{aligned}
$$

Now the numerator and the denominator are both monotone increasing in $y$. Substituting $y$ by .54 in the numerator and by .641 in the denominator, we have

$$
f_{4}\left(3^{1 / 2} y, 1.11, y\right)>\frac{3.11+.93528-1.8316^{1 / 2}}{1.83+1.98^{1 / 2}}>\frac{3.11+.93-1.35}{1.83+1.41}>.8 .
$$

Subcase (b). $u \geqslant w$. Then

$$
d\left(B, C^{\prime}\right) \leqslant\left[u^{2}+(w-y) u+(w-y)^{2}\right]^{1 / 2}
$$


and

$$
\begin{aligned}
\rho^{\prime} \geqslant & \frac{2+w+u-\left(1+y+y^{2}\right)^{1 / 2}}{\left(1+w+w^{2}\right)^{1 / 2}+\left[u^{2}+(w-y) u+(w-y)^{2}\right]^{1 / 2}}=f_{5}(u, w, y) ; \\
N\left(\frac{\partial f_{5}}{\partial w}\right)= & \left(1+w+w^{2}\right)^{1 / 2}+\left[u^{2}+(w-y) u+(w-y)^{2}\right]^{1 / 2} \\
& -\left[2+w+u-\left(1+y+y^{2}\right)^{1 / 2}\right] \\
& \cdot\left[\frac{1+2 w}{2\left(1+w+w^{2}\right)^{1 / 2}}+\frac{u+2(w-y)}{2\left[u^{2}+(w-y) u+(w-y)^{2}\right]^{1 / 2}}\right] \\
< & \left(1+w+w^{2}\right)^{1 / 2}+\left[u^{2}+(w-y) u+(w-y)^{2}\right]^{1 / 2}-(1+w+u-y) \\
& \cdot\left[\frac{1+2 w}{2\left(1+w+w^{2}\right)^{1 / 2}}+\frac{u+2(w-y)}{2\left[u^{2}+(w-y) u+(w-y)^{2}\right]^{1 / 2}}\right]<0
\end{aligned}
$$

since

$$
\begin{aligned}
& \frac{2\left(1+w+w^{2}\right)-(1+w+u-y)(1+2 w)}{2\left(1+w+w^{2}\right)^{1 / 2}} \\
& \quad \leqslant-\frac{(u-y)(1+2 w)}{2\left(1+w+w^{2}\right)^{1 / 2}}<-\frac{u-y}{2} \leqslant-\frac{u-1}{2}
\end{aligned}
$$

and

$$
\begin{gathered}
\frac{2\left[u^{2}+(w-y) u+(w-y)^{2}\right]-(1+w+u-y)(u+2 w-2 y)}{2\left[u^{2}+(w-y) u+(w-y)^{2}\right]^{1 / 2}} \\
\leqslant \frac{u^{2}-u}{2\left[u^{2}+(w-y) u+(w-y)^{2}\right]^{1 / 2}} \leqslant \frac{u-1}{2} .
\end{gathered}
$$

Furthermore,

$$
\begin{aligned}
N\left(\frac{\partial f_{5}}{\partial u}\right)= & \left(1+w+w^{2}\right)^{1 / 2}+\left[u^{2}+(w-y) u+(w-y)^{2}\right]^{1 / 2} \\
& -\left[2+w+u-\left(1+y+y^{2}\right)^{1 / 2}\right] \frac{2 u+w-y}{2\left[u^{2}+(w-y) u+(w-y)^{2}\right]^{1 / 2}} \\
\geqslant & \left(1+w+w^{2}\right)^{1 / 2}+\left[u^{2}+(w-y) u+(w-y)^{2}\right]^{1 / 2} \\
& -\left[2+w+u-\left(1+y+y^{2}\right)^{1 / 2}\right] \\
> & 1+w / 2+u+(w-y) / 2-(2+w+u)+1+y / 2=0 .
\end{aligned}
$$


Since $u \geqslant \max \left\{w, 3^{1 / 2} y\right\}$, it suffices to show

$$
f_{5}(1.11,1.11, y)>.8 \text { for } 3^{1 / 2} y \leqslant 1.11
$$

and

$$
f_{5}\left(3^{1 / 2} y, 1.11, y\right)>8 . \quad \text { for } 1.11 \leqslant 3^{1 / 2} y \leqslant 3^{1 / 2} .
$$

But $f_{5}=f_{4}$ for $u=w$, hence

$$
f_{5}(1.11,1.11, y)=f_{4}(1.11,1.11, y)>.8 .
$$

Therefore we need only prove, for $.64<1.11 / 3^{1 / 2} \leqslant y \leqslant 1$,

$$
\begin{aligned}
f_{5}\left(3^{1 / 2} y, 1.11, y\right) & =\frac{3.11+3^{1 / 2} y-\left(1+y+y^{2}\right)^{1 / 2}}{3.3421^{1 / 2}+\left[\left(4-3^{1 / 2}\right) y^{2}-1.11\left(2 \cdot 3^{1 / 2}\right) y+1.2321\right]^{1 / 2}} \\
& >.8 .
\end{aligned}
$$

Again, the numerator and the denominator are both monotone increasing in $y$. Hence for $\underline{y} \leqslant y \leqslant \bar{y}$, we will substitute $\underline{y}$ for $y$ in the numerator, and $\bar{y}$ for $y$ in the denominator. For $.75 \leqslant y \leqslant 1$,

$$
f_{5}\left(3^{1 / 2} y, 1.11, y\right)>\frac{3.11+1.299-2.2125^{1 / 2}}{1.83+3.2027^{1 / 2}}>\frac{3.11+1.299-1.49}{1.83+1.79}>.8 .
$$

For $.64 \leqslant y \leqslant .75$,

$$
f_{5}\left(3^{1 / 2} y, 1.11, y\right)>\frac{3.11+1.1084-2.0496^{1 / 2}}{1.83+2.2848^{1 / 2}}>\frac{3.11+1.10-1.44}{1.83+1.52}>.8 .
$$

Thus we have shown in all cases that $\sigma(V) / \mu(V)>.8$ and the Theorem follows by induction.

Acknowledgement. The authors wish to thank F. R. K. Chung, M. R. Garey, R. L. Graham, H. O. Pollak and J. F. Weng for helpful comments.

Note added in proof. Recently Chung and Graham announced that $\rho>.8241$ by using computer-assisted computations.

\section{REFERENCES}

1. F. R. K. Chung and F. K. Hwang, A lower bound for the Steiner tree problem, SIAM J. Appl. Math. 34 (1978), 27-36.

2. E. N. Gilbert and H. O. Pollak, Steiner minimal trees, SIAM J. Appl. Math. 16 (1968), 1-29.

3. R. L. Graham and F. K. Hwang, Remarks on Steiner minimal trees. I, Bull. Inst. Math. Acad. Sinica 4 (1976), 177-182.

4. Z. A. Melzak, On the problem of Steiner, Canad. Math. Bull. 4 (1961), 143-148.

5. H. O. Pollak, Some remarks on the Steiner problem, J. Combin. Theory Ser. A 24 (1978), 278-295.

Institute of Applied Mathematics, Academy of Science, Beijing, China

Bell Laboratories, Murray Hill, New Jersey 07974 